\title{
Designing Clinical AAC Tablet Applications with Adults who have Mild Intellectual Disabilities
}

\author{
Ryan Colin Gibson \\ Uni. of Strathclyde \\ Glasgow, UK \\ ryan.gibson@strath.ac.uk \\ Mark D. Dunlop \\ Uni. of Strathclyde \\ Glasgow, UK \\ mark.dunlop@strath.ac.uk
}

\author{
Matt-Mouley Bouamrane \\ Uni. of Edinburgh \\ Edinburgh, UK
}

Matt.Bouamrane@ed.ac.uk

\author{
Revathy Nayar \\ Uni. of Strathclyde \\ Glasgow, UK \\ revathy.nayar@strath.ac.uk
}

\begin{abstract}
Patients with mild intellectual disabilities (ID) face significant communication barriers within primary care services. This has a detrimental effect on the quality of treatment being provided, meaning the consultation process could benefit from augmentative and alternative communication (AAC) technologies. However, little research has been conducted in this area beyond that of paper-based aids. We address this by extracting design requirements for a clinical AAC tablet application from $\mathrm{n}=10$ adults with mild ID. Our results show that such technologies can promote communication between general practitioners (GPs) and patients with mild ID by extracting symptoms in advance of the consultation via an accessible questionnaire. These symptoms act as a referent and assist in raising the awareness of conditions commonly overlooked by GPs. Furthermore, the application can support people with ID in identifying and accessing healthcare services. Finally, the participants identified 6 key factors that affect the clarity of medical images.
\end{abstract}

\section{Author Keywords}

Intellectual Disabilities; Primary Health Care;

Augmentative and Alternative Communication;

Accessibility; Mobile Applications

\section{CSS Concepts}

- Human-centered computing Accessibility

technologies • Human-centered computing $\sim$ User centered design $\cdot$ Social and professional topics $\sim$ Health information exchanges

\section{INTRODUCTION}

Medical professionals are increasingly overworked [1, 2], which impacts their ability to seek out further training opportunities to enhance their skillset. This is problematic in the context of healthcare for people with intellectual disabilities (IDs), since medical schools often deliver highly variable and somewhat limited content regarding this population [3]. As a result, practitioners are apprehensive

Permission to make digital or hard copies of all or part of this work for personal or classroom use is granted without fee provided that copies are not made or distributed for profit or commercial advantage and that copies bear this notice and the full citation on the first page. Copyrights for components of this work owned by others than ACM must be honored. Abstracting with credit is permitted. To copy otherwise, or republish, to post on servers or to redistribute to lists, requires prior specific permission and/or a fee. Request permissions from Permissions@acm.org.

CHI 2020, April 25-30, 2020, Honolulu, HI, USA

(C) 2020 Association for Computing Machinery.

ACM ISBN 978-1-4503-6708-0/20/04 ..\$15.00 https://doi.org/10.1145/3313831.3376159 about their ability to treat patients with ID and are unaware of their specific health and communication needs [4].

This has a negative impact on both the length and quality of life of such patients. For example, a 2013 inquiry into the premature deaths of people with ID concluded that circa $50 \%$ of the 247 cases examined were avoidable, with $27.5 \%$ of these directly attributable to better quality health care [5]. Furthermore, each patient suffered from an average of five long-term / treatable conditions at their time of death, many of which are straightforward to diagnose and cure e.g. constipation was found in $37 \%$ of these cases.

Communication has been identified as a significant barrier to accessing effective health services for people with ID. [5-9]. Yet despite its importance in providing optimal care [10], medical professionals often find it difficult to adjust their consultation styles to cater to the complex needs of patients with ID $[4,8,9]$. Given the intense workload pressures previously described [1, 2], General Practitioners (GPs) are unable to invest the time required to increase their knowledge in this area. Digital technologies therefore become an important resource for supporting effective communication between GPs and the ID population. However, their use in this context remains vastly underresearched in comparison to other domains such as [11-13].

We address this gap by investigating the implementation of tablet applications to promote communication between patients with mild ID and GPs. This was approached from the perspective of patients since their crucial views have been omitted in other studies [14-16]. Three user-centered design workshops (UCD), involving ten adults with mild ID, were conducted to decipher the participants requirements for the proposed tablet application, along with their current use of alternative and augmentative communication (AAC) technologies. Such technologies are used to enhance an individual with disabilities capacity to communicate by offering those who cannot speak a platform to convey their needs (alternative), or by supplementing the vocabulary of those who can (augmentative) [17]. The results obtained enabled us to answer the following two research questions:

RQ1: What range of technologies are patients with mild ID using to assist them in communicating with GPs?

RQ2: What do patients with mild ID require from a tablet application to support them in communicating with a GP? 
We therefore contribute to the field of HCI by establishing a set of design requirements to assist in the future development of clinical AAC applications for adults with mild ID. In addition, we provide guidance on the use of mobile technologies to improve healthcare services for people with communication impairments, by building on the findings of $[12-15,18]$.

\section{BACKGROUND}

In this work, we consider ID using the World Health Organization (WHO) definition [19]: "[they have] a significantly reduced ability to understand new or complex information and to learn and apply new skills (impaired intelligence). This results in a reduced ability to cope independently (impaired social functioning), and begins before adulthood, with a lasting effect on development." The severity of an individual's ID can range from mild to severe. People with mild ID can generally conduct everyday tasks without support but tend to struggle with those that are unfamiliar or more complex. Consequently, we believe this population could benefit significantly from a clinical AAC application, since such technologies can augment their vocabulary and language skills whilst they are interacting with a GP. This view is also supported by the medical professionals, support workers and academics included in the literature by Gibson et al. [14-16]

\section{Non-Digital Communication Aids}

Paper-based AAC resources are a common form of support utilized by people with ID. As such, several of these have been adapted for use within the clinical domain.

\section{Patient Passports}

Patient passports [20] encapsulates an individual's personal profile (e.g. their environmental, medical or communication needs) to support practitioners in providing patient-centered care. Nevertheless, to be used effectively, medical professionals must possess the skills required to meet the complex needs of a patient. In addition, Northway et al. [11] found that the availability of passports, and the content included within them, varied widely across health centers. Consequently, their overall benefit is reduced as there is no guarantee that the information provided will be utilized effectively by staff, or whether the same passport can be used throughout the healthcare system.

\section{Talking Mats ${ }^{\mathrm{TM}}$}

Talking Mats ${ }^{\mathrm{TM}}$ [21] is a resource utilized by people with communication impairments to discuss difficult subjects in greater depth. The user provides their opinion by placing images (relating to options) under the relevant section of a visual scaled embedded within a textured mat. Bell and Cameron [22] used the Talking Mats ${ }^{\mathrm{TM}}$ framework to determine the mental health status of a patient with mild ID. This enabled the patient to supply their views whilst avoiding the social pressures that may arise when directly interacting with a health professional [22]. Consequently, the quality of the information provided increased, which led to a successful diagnosis being carried out. However, the framework requires a large amount of modification for use within different contexts, due to its reliance on images.

\section{Digital Communication Aids}

The use of digital communication aids to support patients with ID in other areas of the healthcare system has also been investigated extensively.

\section{Dentistry}

Menzies et al. [18] developed a computer-based tool to support patients who have ID in communicating with dental practitioners. They found that the aid could improve consultations in three main ways: (1) presenting images and videos of dental procedures prior to the appointment, to increase the patient's understanding of what may occur; (2) providing staff with access to the patient's personal preferences to assist in building a rapport with the individual; and (3) presenting information in the format most appropriate to the patient's needs.

\section{Secondary Care}

Prior et al. [12, 13] also placed emphasis on the use of patient passports when developing an extended, digital version for secondary care services. Their system is centered on customization to ensure it is appropriate to the patient's clinical and accessibility needs. As such, the patient may select from a variety of input methods and is only required to answer a subset of the questionnaire, based on aspects such as their age and gender. 3 image sets were also implemented to increase the user's comprehension of the question being asked, along with the ability to switch between these sets at any time [13]. This system therefore provides several advantages over the paper-based resource since it prioritizes certain aspects of an individual's care and stores the emerging results in a centralized location.

\section{Mental Health}

In the work most similar to our own, Boström and Eriksson explored the use of tablet technologies to support children with ID in self-reporting their psychological health [23]. They employed user-centered design techniques to develop a 43-question survey across five topics of an individual's mental health. A quantitative study was used to measure the accessibility of this questionnaire, with 109 of the 113 participants involved able to answer all questions without support and with limited signs of response bias [24].

Finally, to ensure the results of the questionnaire matched the views of the user, a mixed methods study was carried out with ten children who have mild ID [25]. Boström and Broberg found a good level of agreement between these results and the views provided by the participants during a series of semi-structured interviews. Nevertheless, some of the more negative experiences described were not picked up by the questionnaire, and this was attributed to such aspects being omitted from the app [25].

Boström et al's work [23-25] indicates that a digital questionnaire can be an accessible resource for extracting reliable data from people with ID. This data may then be 
used to open up a discussion on how the patient is feeling and ultimately lead to medical professionals carrying out better informed care decisions [24]. As such, there is ample opportunity to explore how these technologies may be employed within primary care, since the aforementioned paper-based aids [11, 20-22] have several limitations.

\section{METHODOLOGY}

To ensure the proposed tablet application is developed systematically, we have employed the Medical Research Council's framework for complex interventions [26]. The study presented in this paper adheres to the "Development" phase since its focus is on establishing how the application can fit into and improve current practice. Determining whether improvements have been achieved at multiple stages throughout a project is well-suited to highly heterogeneous populations, as it may be initially difficult to gather requirements from such stakeholders. In addition, the framework is widely accepted throughout the medical domain. Therefore, developing the app using this approach increases the likelihood that it is usable by people with mild ID and is embedded within current practice.

How the app can improve primary care consultations was established by extracting design requirements from ten adults with mild ID across three UCD design workshops. The design tasks implemented are described in the "Procedure" subsection and aimed to address three aspects deemed crucial to the implementation of AAC technologies: (1) their overall functionality (2) the layout and design of each screen; and (3) images that capture the options presented, since people with ID have difficulty understanding complex concepts and have lower literacy skills than the general population [12-16, 18, 27-30].

\section{UCD Workshops - Participants}

Four ID charities throughout Scotland were contacted via email and telephone to assist in identifying participants who adhered to the inclusion criteria for the study. These criteria were comprised of: adults aged between 18 and 60 (to reduce the presence of age relate cognitive diseases) who have mild ID only; individuals who can communicate via speech (including with the use of AAC devices) and can understand verbal partners; and those with the visual capabilities to process images.

Based on the recommendations of the experts involved in [16], the charities were asked to recruit between 4 and 6 participants per workshop. Easy read information sheets were then disseminated to potential candidates to enable them to gain an understanding of what the workshop entails, with an in-depth version being sent to their caregiver to promote discussion. Potential participants were invited to take part if they could demonstrate their ability to provide informed consent, by answering the six questions proposed by Horner-Johnson \& Bailey [31].

\begin{tabular}{c|cc} 
Participant ID & Gender & Age \\
\hline $1.1,1.2$ & F, M & 41,29
\end{tabular}

$$
\begin{array}{c|cc}
2.1 \text { to } 2.5 & \text { M, M, F, F, M } & - \\
3.1, \text { to } 3.3 & \text { F, F, M } & 28,29
\end{array}
$$

Table 1. A description of the participants demographics.

Three UCD workshops were carried out between the months of August 2018 and July 2019 in the cities of Glasgow and Edinburgh. Four individuals consented to take part in workshop one; however, two dropped out unexpectedly on the day. Five participated in workshop two, with a further one dropping out and three took part in workshop three (see table 1 for demographics). Each had an unspecified range of disability that adhered to WHO's definition of mild ID. Further information regarding their etiologies was not collected as we felt it was inappropriate to ask the participants about the nature of their disability. The final $\mathrm{n}$ size therefore adhered to the recommendations of Doyle to gain a diversity of views that lead towards saturation [32]. One charity facilitator was present during each workshop to assist the lead author in solving any challenges that arose.

\section{UCD Workshops - Procedure}

Before each workshop commenced, the participants were briefed on the structure of the study and had any concerns addressed by the lead author. They were then required to sign an easy read consent form, before engaging in an icebreaker session to help them feel at ease with their peers. The four tasks shown in Figure 1, were then presented for completion, with these tasks previously being adjusted (with the help of experts in ID) to cater to the accessibility needs of the participants [16].

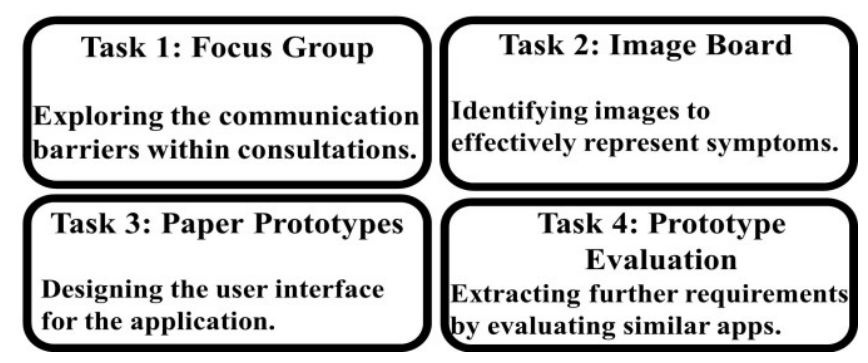

Figure 1. The tasks completed by participants with mild ID.

Task one consisted of a focus group to explore the participants experiences with primary care and determine how technology can support them within this environment. The questions presented were developed using accessible language guidelines and were primarily open-ended to promote discussion. The topics centered on 4 main themes: preparing for consultations; positive and negative communication encounters with GPs; their use of AAC devices; and how technology can improve consultations. Additionally, the key concepts discussed were captured in real-time via the use of sticky notes (like [33]), and subsequently presented in full view of the group. This enabled them to challenge any misconceptions made by the lead investigator and assisted in overcoming short-term memory impairments. 
During task two, the participants were required to develop two images boards [33] that captured their opinions on the clarity of medical images. Each individual first critiqued a set of pictures before separating them into two categories: those that accurately represent the symptom conveyed; and those whose meaning is more obscure. A group discussion then occurred on why some images were clearer than others, before being placed on the appropriate board. All images were accompanied by a short textual description to ensure the participants knew what it was trying to depict.

Task three involved developing a paper prototype to establish the patient's views on how the proposed application should function. They were required to place mock-up objects onto a paper representation of a tablet and position them according to their needs. These objects included general usability features, such as skip buttons, as well as those more specific to the proposed application. Blank objects were also included to allow participants to incorporate elements unforeseen by the authors.

In task four, the participants were required to evaluate a digital prototype of the application, developed using guidelines disseminated by experts in ID. [14, 15] This enabled us to discern requirements that had not been identified during task three (without initially biasing the participants views), since there is evidence to suggest that people with ID become more engaged when interacting with digital prototypes [34]. They had to select various symptoms using the application (without assistance) and any areas of uncertainty were noted. Once completed, the lead investigator prompted for an explanation of the participants thoughts during their uncertainty, as well as the areas of the application they liked and disliked.

Comfort breaks were administered between each task, with the participants being reminded of their right to withdraw during this time frame. Each workshop lasted approximately 3 hours, at which point the participants were debriefed on the initial results obtained and reimbursed for the travel expenses they had incurred. They also received a $£ 5$ lunch voucher for their participation. All studies were conducted under ethical approval from the Department of Computer and Information Sciences ethics committee, University of Strathclyde (ID: 915).

\section{UCD Workshops - Analysis}

Tasks one to four were audio recorded with participant consent and transcribed verbatim by the lead investigator to familiarize themselves with the data. Task one was then subjected to a framework analysis [35], with an initial thematic framework being developed by the first author using the concepts captured within the aforementioned sticky notes. This framework was used to tag the transcripts, with additional codes being developed where necessary. Author four subsequently reviewed the tagged transcripts and any discrepancies were resolved, at which point similar codes were grouped together to form themes. Consequently, a structured summary of the barriers faced by the participants within the clinical context, as well as the strategies used to mitigate these barriers, was produced.

Task four underwent a similar analysis process except that the initial framework was developed by the lead author during the familiarization stage. This enabled us to gauge aspects of the prototype that were accessible to adults with mild ID, as well as those that may hinder the communication process.

During task two, the participants were required to group logically related images together whilst forming the (un)clear boards. For example, images that depicted the wrong facial expressions were placed in a similar area on the unclear board. The groupings that emerged were then tagged using phrases suggested by the participants and subsequently combined to form overarching themes. These themes enabled us to provide guidance on the development of medical images for people with mild ID.

During task three, the lead investigator proceeded to tag each distinct feature produced by the participants. These features were then transferred to a spreadsheet, which included a column detailing the number of workshops in which they were discussed. As such, we were able to sort it by the frequency column, thus giving developers an idea of the requirements prioritized by adults with mild ID.

\section{RESULTS}

We will now discuss the primary results that emerged across the four tasks. The requirements in which these results translated to may be found in Tables 2 and 3 .

\section{Task One - Focus Group}

Throughout the focus groups the participants discussed several barriers they face when attempting to access effective healthcare services. In addition, they introduced the various technical and non-technical strategies used to mitigate such barriers.

\section{Access to Healthcare Services}

The participants experience with primary care practices varied widely. For example, Participant (P) 2.1 had only attended in recent months to undergo their yearly ID health check, whilst others had made regular appointments to manage chronic conditions or mental illnesses (Ps 1.2, 2.1, 2.3, 3.2 \& 3.3). Despite this range, all reported experiencing barriers when accessing services from their GP, the most prevalent of which was the availability of appointments. P2.3 revealed that it can take up to 3 weeks to arrange a consultation within her practice, leading to detrimental effects on both their mental health and ailment: "For a normal appointment you've got to know three weeks in advance what you've got... if there's not an appointment for 3 weeks you could be worrying unnecessarily till you find out whether it's something serious or whatever."

This waiting period is prolonged even further if the patient requests to be treated by their preferred GP within their practice - see section "Practicing GP." Nevertheless, due to 
the seriousness of their condition, Ps 1.1 to 2.3 have had to settle for treatment by doctors they are unfamiliar with. This has led to P1.1 falsely claiming that their condition is an emergency to ensure they receive a timely appointment with their favored practitioner.

The participants also reported a large variance in the organizational procedures used to disseminate information and book appointments. Furthermore, these procedures were often static, with employees unable to adjust the methods used to meet the needs of a patient with ID, as described by P3.3: "I got my [diabetes] diagnosis over the phone which more or less just didn't work. There was no face to face sort of contact, and it ended up me just basically ignoring my diabetes for quite a few years. There wasn't really much in the way of clear communication."

Finally, the participants also indicated that they were not always aware of their need to see a doctor and instead rely on family members to facilitate this process. Nevertheless, they often withhold crucial information regarding their health, as they do not want to overburden their loved ones: P1.2: "My family do a lot of caring work, so to give them information that I'm not well then I'm probably going to feel a little bit over[whelmed]..A family member says "right you need a doctor" and that's about it [for preparation]."

\section{Practicing GP}

The participants had conflicting views regarding the quality of care received from their GP, which often leads to them dealing exclusively with certain doctors. Their complaints largely centered on the lack of adjustments being made by a medical professional to accommodate their specific needs. Terminology was a major factor in this, with the GP regularly employing complex terms, or language inappropriate to the patient's level of ID: P3.2: “Because I speak so well doctors think that I understand more than what I do... and I'm like "what? Can you simplify that?"," P2.3: "If they see the word learning difficulty, they just think that's it, obviously they've got the mental capacity of a two-and-a-half-year-old so I'll just talk to her like that when there is different levels."

Furthermore, the participants felt that some medical professionals lacked empathy towards their situations. This meant that an insufficient amount of time and effort was expended on diagnosing the health complaints made, which may ultimately lead to dire consequences: P2.3: "I just felt that they weren't interested. They were running late, and they were just wanting me in and out the door. They weren't interested in sort of me or what was wrong with me. Which luckily, if I was somebody that didn't have kids that was on the brink, that could have serious repercussions"

In contrast, the positive experiences discussed by the participants centered on their preferred GPs ability to meet their complex needs. Language was also a major part of this, with the medical professional being aware of the best way to communicate with the patient, whilst breaking complex concepts into simpler terms: P1.2: “They don't use the complicated language or if they do they then say it in simpler terms for you as well." P2.3: "He sort of asked loads of questions but, like, it was simple short questions, it wasn't a case of big long winded [sentences]."

Being treated by the same doctor also helped the participants to establish a relationship with a figure who is often seen as authoritative. This assists in easing anxiety issues and in turn enables the patient to be more open about their health conditions. Additionally, people with ID are more prone to developing a vast range of diseases than the general population [5]. As such, their medical histories can become complex meaning it may be difficult for GPs unfamiliar with the patient to perform a correct diagnosis. P2.5: "If you get to use the same doctor, you get friendly with them and they get friendly with you, so you are able to speak to them more." P3.2: "I can go to the GP without my mum because she knows me that well. I've got the confidence to not go with support...I try and see the same doctor for anxiety reasons but also for the doctor's sake cause my history is so complicated."

Time

The amount of time afforded for appointments was also reported to be a major barrier that effects the quality of consultations. Less than half of the participants (P2.3, 3.1, $3.2 \& 3.3$ ) would adequately prepare for an upcoming consultation and those who would, found it difficult to cover all aspects they wished to: P3.1: "Sometimes I have questions, but the problem is you don't get very long in the GP...You try to get all those questions [answered] in the ten minutes, it's quite a struggle."

In addition, the participants believe that time constraints prevent medical professionals from thoroughly exploring all routes leading to the cause of a symptom, including familiarizing themselves with the patient's history: P2.4: "You go in and you talk about what you want and then they get up and open the door and you're just told that's it, it's time to leave"P1.2: "They just don't have time to read your record. They go in and they say, "well what's wrong with you” [participant] "Oh I've got...." [doctor] "oh we'll give you Co-dydramol.","

Consequently, patients with ID could benefit from double appointments. Yet when probed on this, only P3.2 revealed that they regularly use such an option, with the rest unaware of their right to do so. This suggests that practices are not adhering to international guidelines on consultation length e.g. [36]; nevertheless, there were some GPs willing to overextend on appointments to ensure their patients had all concerns addressed: P1.2: "If I'm having trouble with my diabetes then the doctor that I go to see in the practice, he makes time. He'll deliberately go behind his schedule so that he can make sure that everything is back okay and, you know, figure out why I'm having trouble with my diabetes." 


\section{Support}

Surprisingly, the majority of participants reported that they attend GP appointments with support workers, despite previously indicating they value their privacy. This may suggest that their willingness to share personal issues with paid caregivers differs from that of their family members to limit the amount of stress placed on loved ones. The primary responsibility of the caregiver was to act as an intermediary between the patient and GP to ensure the stakeholders understood what was being communicated. This was particularly true during appointments of a complex nature. P2.3: As soon as they found out I had learning difficulties and I started taking my support worker they explained more to my support worker. So even if I didn't get it then and there, when I went away, she's going to explain it in a way I would understand.

In contrast, P2.4 and P3.2 reported having negative experiences, as they felt their own views had less value in comparison to the caregivers. P3.2: [When my] mums tried to support me it's like "well have you asked this or what can we do for this" and I'm like just sitting listening. If I'm on my own I will have the guts to sort of say "what's this or why are we doing this or would this be helpful," like I will ask the questions. I think my mum sort of has the best intentions but she kind of overrules a little bit. Finally, P2.2 requires support during consultations but cutbacks in funding meant this was not feasible.

\section{Technological Aids}

The participants reported a high usage of memory aids (pen and paper) to support them in recalling facts that require further scrutiny out with the consultation. Strikingly, this contrasted with their use of AAC technologies, with only P3.2 having utilized a form of patient passports in the past.

Nevertheless, all participants felt that a digital aid could assist both themselves and patients with other/more severe disabilities to better prepare for a consultation, thus enabling them to communicate effectively with GPs: P1.2: "It [the app] should gather symptoms and then send them to the GP. [Because] for us with mild learning disabilities, sometimes we find it difficult to describe symptoms. Thinking about physical disabilities it's also a good idea because with a tablet it [would] quite literally be a case of boom into the doctors, right this is what it is...right that's a chest infection, here's some antibiotics."

One major barrier to the adoption of such aids, however, is this populations familiarity with tablets, since just five of the participants own or use a tablet on a regular basis. On the other hand, all had access to a smartphone with P3.3 stating that these devices are more accessible, in terms of availability, than tablets.

\section{Task two - Image Boards}

Previous literature has shown the importance effective imagery has on the use of AAC devices by people with ID e.g. $[13-15,18]$. Nevertheless, there has been little exploration into the factors that ensure medical images are identifiable to this population. Consequently, the participants were required to evaluate three image sets real-life photographs, simplistic black and white drawings and colored cartoons - and subsequently form two image boards based on the artefacts' clarity. The key elements that influenced their selections will now be discussed.

\section{Facial Expressions}

The emotions expressed by an individual had a significant impact on the clarity of an image. For example, two of the three workshops originally concluded that the man in Fig 2.1 could not be experiencing pain since his facial expressions alluded more to happiness. Moreover, the participants in workshop two were unable to associate the emotion of sadness with the image shown in Fig. 2.2: P2.4"I think he was in a dream or something. P2.3: "He looks like he's playing with his tablet...I think I'd like tears, like maybe one of those things with like tears or something." P2.3 suggested that the inclusion of tears would improve this image's clarity since it may demonstrate that the person has been crying. Consequently, these views may imply that it is more appropriate to capture the extremity of an emotion to ensure it is understood by the ID population as intended.

\section{Body Position}

There were multiple instances in which the position of the body was considered the most important aspect of an image. P1.2 was generally able to grasp the meaning of Fig. 2.1. However, they questioned the location of the character's head since he associated the action of looking up with being in pain. Furthermore, the participants in workshop two failed to agree upon the clarity of the image displayed in Fig. 2.3: P2.1: "He's sort of dozing off there he can't keep his eyes open. P2.5: “No, he's standing up." P2.3: You couldn't sleep standing up. It's a weird one."

Despite the facial expression and presence of " $z$ ' $s$ " making the image clear to other participants, P2.3 and 2.4 could not see past the character standing up. Consequently, they were more inclined to select images that depicted the character lying in bed - an action they found more natural for this scenario. Finally, P2.3 and P2.4 were able to recognize that the woman in Fig. 2.4 was in distress but felt that the area of pain was emanating from her cheek due to the position of her hand. As such, designers must ensure that their images naturally capture the body language experienced by an individual suffering from the condition displayed.

\section{Color}

Color was shown to both affect the clarity of an image and differentiate between similar types of pain. For example, P3.2, who is short sighted, failed to identify that the man in Fig. 2.5 was sitting in a restroom: P3.2: "I had to really look closely to see because he's wearing a white outfit on a white toilet so I couldn't even identify the loo. I was thinking more headache because he's holding his head." This image may therefore have benefited from the use of 


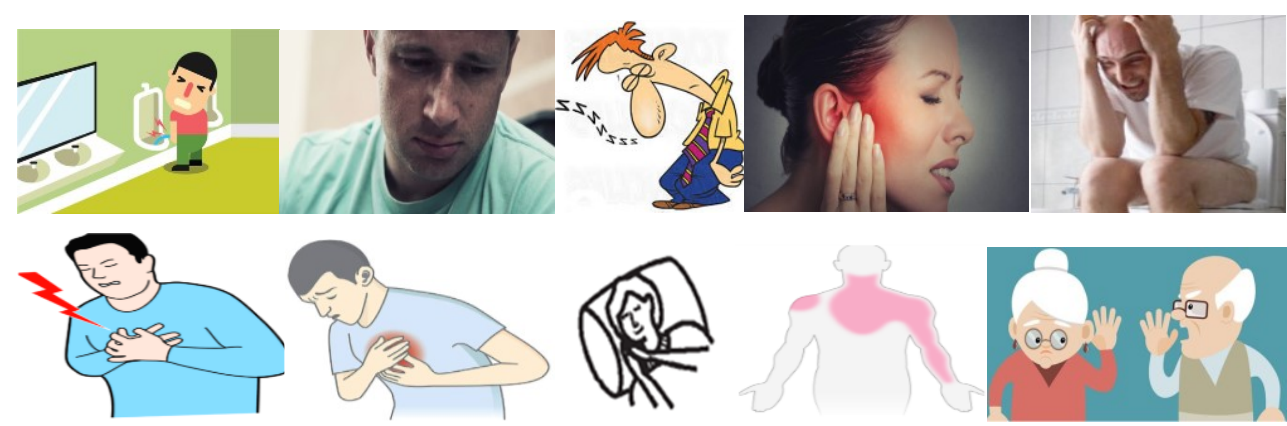

Figure 2: A subset of images critiqued by the participants during Task 2.

contrasting colors to enable people with visual impairments to recognize its finer details.

P1.2 also raised this point and stated that the black and white pictures are appropriate for those who are color blind but may be less aesthetically pleasing for those with normal vision. Additionally, the participants preferred using color (as opposed to other methods such as Fig. 2.6) to indicate the area and intensity of pain, like that shown in Fig. 2.7. In general, they found warm colors such as red or peach to indicate more intense pains with cooler colors such as green and blue representing a numb or tingling feeling.

\section{Lifelike}

The participants routinely found the real-life photographs to be clear, as they reminded them of conditions they had previously experienced. This was due to the amount of detail that may be conveyed: P1.2: "It says what it needs to say but to me the one that we've picked over there said it more...Sometimes actually seeing the physical side of it and the emotional side of it does work better than the drawing."

Moreover, the participants felt that even less detail could be captured by the black and white drawings. For example, in Fig. 2.8 they found it difficult to establish whether the woman's eyes were open. As a result, P1.2 explained that the black and white image sets may be more appropriate for users with severe ID but are too simplistic for their own abilities. This was also true for those drawings that captured the outline of a body (Fig. 2.9) since no distinguishable features were included.

\section{Graphic}

During each of the workshops, the participants found at least one image to be too graphic to include in the app. P3.2 suggested that color drawings should be used to capture these concepts as they are less realistic and may be altered to obscure the graphic nature of a condition: "I think that is a little bit too real looking. Whereas the other one [colored drawing of someone being sick] that's like a green color, it's a little bit of a distraction."

\section{Personalization}

The final concept discussed by the participants in relation to the images reviewed was personalization. There were multiple instances in which a participant hesitated to declare that an image was clear since the traits of the character displayed were completely different to their own. This included both gender (Fig. 2.1) and age (Fig. 2.10): P2.5: "I prefer this one cause that's just showing you male and that one's showing both." P1.1: "I liked the picture, but I didn't pick that one cause it just says that old people are deaf whereas young people can [also] be deaf."

In addition, images may have multiple meanings based on the communication system the user is familiar with: P1.2: "People with more severe learning disability who are used to PECS [37] will pick out things like the person with the lines round him is cold, the person with the arrows is dizzy. They'll pick that out because that's what they're used to." Consequently, this suggests that AAC technologies must provide the functionality to support users in switching between multiple image sets based on their own needs.

\section{Task Three - Paper Prototypes}

During the development of the paper prototypes, the participants identified a plethora of design requirements to be embedded in clinical AAC tablet applications. These are summarized in Table 2 and generally fit into four themes.

\section{Pre Health Questionnaire}

In advance of providing medical information, the participants requested two features to assist them in attending the consultation. Ps 2.3 to 2.5 revealed that they had issues remembering the exact details of an upcoming appointment and could therefore benefit from a screen that displays this information $P 2.5$ : "A reminder about your doctor, when you've got to go. Cause quite a lot of people, they do forget about their appointments. Now if they have something there to remind them about it [that would be helpful]." The time and location of the appointment, along with the practicing GP were considered to be the most important aspects within this process.

During workshop three, the participants also discussed the difficulties they have in both contacting and accessing appropriate services: P3.2: "If you put in like your post code that way it can identify [your] closest GP. Near me I've got 3 different GPs, so it can direct you to the [details of the] nearest one. You could have sort of like the top 5 [services] dentist, mental health, hospital, $A \& E$, and GP...A lot of us do need public transport because I can't get to <anon> easily. Maybe a little corner bit on that 
front page to say "here's the link to these forms of transport.","

Hence, the app could enable them to identify the most appropriate local service to treat their current condition, ranging from dentistry's to accident and emergencies. In addition, P3.2 felt that it was paramount to provide public transport links for these services thus breaking their reliance on caregivers to gain access to the healthcare system.

\section{Health Questionnaire}

All participants agreed that the most effective way to improve communication with a GP was to supply them with a list of pre-selected symptoms. This should be achieved via an accessible questionnaire whose structure follows a hierarchical route. First, a body part causing the user distress, or the primary symptom of a common condition, should be identified. Further options related to that selection should then be explored: P3.2: "Maybe you had something that said like different parts of your body. So head, chest, arms, legs, you have the headings like that and [then] you go into the subheadings for like symptoms."

P3.2 suggested that the body parts and primary symptoms (e.g. weight loss) could be displayed in a color coordinated, textual list. Nevertheless, this may be inappropriate for those who are illiterate or require more visual methods of displaying information. As such, the three workshops suggested an alternative approach by first presenting an image of the body to enable the user to tap on the area causing them pain. The app would then move on to displaying sub-symptoms in an accessible format (see "Interaction Modalities") or if the person was not in pain display the primary symptoms described previously. A potential model to achieve this questionnaire structure is discussed in [38].

\section{Post Health Questionnaire}

Once the questionnaire is complete, the participants requested that the results be displayed in a single screen using the modalities discussed in the next sub-section. As such, the stakeholders involved in the consultation may refer to this information when elaborating on their views. To facilitate this process, a save and review feature must be implemented, as well as an option to print the results.

\section{Interaction Modalities}

All participants discussed the need to capture the information displayed via three modalities. The first, accessible language, entails describing the symptoms and questions in the simplest terms possible. This includes avoiding medical jargon where appropriate; however, such a strategy may not be suitable for patients with visual problems or insufficient literacy skills. Consequently, the option to highlight and play back excerpts must be provided as described by P1.2: "This [audio] button would [first] say [the question] "are you in pain" and then highlight the yes [option before] saying "yes" and then highlight no... Three separate buttons [for each option] would make it more difficult for somebody with a LD. " In addition to speech and text, the participants believe that imagery would help them to understand the more complex symptoms. This was also true for representing the function of buttons, as described by P1.2: "See thinking about it the guy with the speech bubble would probably be better cause that's saying that it [audio button] can read it for you."

\section{Requirement}

Personal profiles must be facilitated to enable features such as saving symptoms in a patient history. $(*)$

The application should initially determine whether the patient has a problem with a body part or some other common condition e.g. diabetes. (*)

Question sets should form a hierarchy with selected symptoms leading to relevant sub-symptoms. (*)

Patients may select more than one option at a time. Selected options should be highlighted to distinguish them from those unselected. $(*)$

Patient's should have the option to show where their pain is by tapping on an image of the body. $(*)$

A maximum of 4-6 options should be presented at any one time. $(*)$

The language embedded within should follow accessibility guidelines. Medical jargon should be avoided where possible. (*)

Audio playback of text should be supported within all pages. $(*)$

Images should be included to enhance an individual's understanding of a medical condition, as well as an embedded button's functionality. (*)

An accessible list of the symptoms selected should be displayed on completion of the questionnaire. $(*)$

Left (back) and right (forward) arrows should be used to navigate from a page. $(*)$

Patients should be able to view the details of upcoming appointments. (2)

Users should be able to view public transport routes to local services. (3)

The app may be used to manage a list of medication being taken by the user. (3)

Selected symptoms should be saved for future use. The ability to print these results must also be offered. (2)

Color can group logically related items together. (3)

Scrolling should be avoided if possible. (1)

The app should provide access to the user's patient passport if available. (3)

Table 2. Features to be included in medical AAC applications for patients with mild ID. * indicates that all workshops discussed the requirement with 1, 2 and 3 used otherwise. 


\section{Task 4 - Digital Prototype}

In the final task, the participants were required to select symptoms via a digital prototype developed using the requirements discussed in [14, 15]. Fig. 3 shows the main page layout embedded within this prototype, which follows a similar hierarchical procedure described previously. The feedback received during this process will now be discussed and is summed up in Table 3.

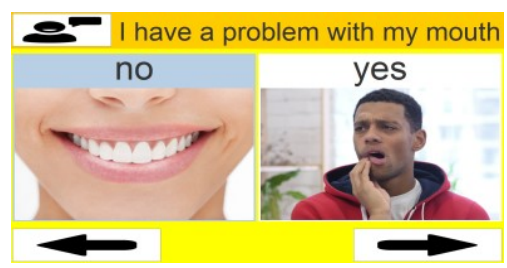

Fig 3. Primary Screen included in the prototype.

\section{Tutorial Screen}

Despite the symptom selection process being similar to that identified in the previous task, workshops 2 and 3 were initially unable to grasp the concept of the questionnaire hierarchy. Once it was explained that answering yes to a primary question would lead to its sub-questionnaire being presented, the participants were able to progress through the application without support. As such, multiple participants suggested that it would be necessary to include a tutorial to enable users to familiarize themselves with the app: P3.2. "That [the questionnaire hierarchy] I think you would have to like explain a little bit beforehand because that was a bit confusing there until you got to that point."

\section{Customizing Features}

Yellow was utilized as the primary background color to accommodate for users who experience dyslexia [39]. Nevertheless, the participants felt that a range of colors may be more appropriate for other medical conditions or to simply meet individual preferences. As such, they requested the ability to customize the color schemes employed. P3.2 also felt that it may be advantageous to customize the voice used to play back text to a more local dialect.

Finally, the lead author anticipated that the utilization of a single image set would lead to accessibility issues for users with ID. Consequently, a feature was developed to allow an individual to switch between three styles of images and this was well received by all participants: P2.3: "I like the fact that you can change it. Some images aren't as clear but then the other ones are a bit clearer, so if you are confused you can change the image and understand [it] better."

\section{Usage of Results}

All participants felt that the app could improve communication by providing a building block of symptoms that may be elaborated on throughout the consultation. This may also empower patients with limited verbal skills, as discussed by participant 2.3: "It sort of would be good to have something like that for, like, people who maybe aren't as good at communicating, that can just point to it and have a limited conversation. Like for me I'm reasonably alright so therefore I can sort of explain reasonably well if I'm not well. Whereas not everybody is like that so having this I think is a good idea cause then you've got the basics, so you just have to polish it up kind of thing. "

One final approach to utilizing the captured results was discussed by the participants in workshop three. They suggested that sending the list of symptoms to the practice in advance of the consultation could assist in overcoming the various access barriers introduced previously: P3.2: "If you could scan that result and send it to your GP and they said, "oh you don't need to come in cause it's [not serious]." So it would also make it beneficial to the actual service of the thing because you don't actually want to go unless you have to." This process would enable the practice to arrange an appointment date relative to the seriousness of the symptoms selected. Consequently, they may also be able to free up consultation times by forwarding on patients with less serious illnesses (e.g. a normal headache) to other services such as a pharmacy.

\section{Requirement}

Users have access to a tutorial on how the app works. (*)

Users can switch between different image sets. $(*)$

Users can customize the color schemes employed. $(*)$

Users can customize the style of voice played back. (3)

Results should be sent to the practice in advance of the consultation to ensure appointments are made within a suitable timeframe. (3)

Table 3. Further features identified by the participants when completing task four.

\section{DISCUSSION}

Many of the barriers identified in task one feature heavily throughout the literature published in the last two decades. For example, time constraints and the lack of adjustments being made to the consultation techniques used by GPs were discussed in $[8,9,40]$. This suggests that despite major advancements being made in the past, there is still a way to go to ensure the equal treatment of patients with ID.

Technology has a major role to play but cannot solve all the barriers previously discussed without wholesale changes being made to the social culture and processes contained within the healthcare system. Trollor et al. [3] demonstrated this when examining the depth of ID training received by medical students in Australia. They found that the students received just 2.55 hours of compulsory training and the elective courses available varied markedly in both quality and length. As such, Trollor et al. concluded that there is " $a$ mismatch between the considerable unmet health needs of people with intellectual disability and the inconsistent teaching within medical schools" [3]. This may be partly responsible for the low levels of confidence reported by medical professionals $[4,41]$ when treating people with ID. 
One aspect of the consultation process that may immediately benefit from the use of technology is communication - particularly when our findings show that AAC devices are vastly underused in this context. We have provided guidance on how to develop these technologies by extracting design requirements from end users. Some of the lessons learned during this process, may also assist in increasing the accessibility of healthcare services for other populations who experience communication impairments.

\section{Overcoming Access Barriers}

The bulk of the literature investigating the use of AAC in healthcare $[12-15,18,20]$ has overlooked the barriers people with ID face when accessing such services e.g. [5, 6, 9]. Nevertheless, the end users involved in our study felt that the proposed app could alleviate access barriers in two main ways. First, it can support people with ID in identifying the most appropriate service to meet their medical needs, along with public transport routes to attend this service. Second, the participants believed that the app could assist them in being treated in a timely manner. This may be achieved by sending a list of symptoms to a practice in advance of the consultation, to enable an appointment to be made in a time period relative to the seriousness of the symptoms.

\section{Providing the Patient's Accessibility \& Medical Needs}

Technology may support medical professionals in administering person-centered care by providing them with information regarding the needs and skills of the patient $[12,13,18]$. P3.2 agreed with this and requested that an individual's patient passport be integrated with the app where available. Nonetheless, the participants also suggested that allowing the GP to have access to a list of pre-selected symptoms could have significant benefits to the consultation, therefore agreeing with the experts involved in [14-16]. This should be facilitated by an accessible questionnaire that first extracts the primary symptom experienced by the user, before exploring further symptoms related to that condition. Such a structure enables the questionnaire to incorporate a wide range of conditions, whilst remaining succinct enough to accommodate for short attention spans [38]. In addition, the questions embedded within should be based on the health demographics of the ID population, meaning the app has the potential to identify conditions commonly overlooked by GPs. The results will be used as a referent throughout the consultation and enable GPs to concentrate on areas of interest for longer, thus alleviating any time constraints present.

\section{Customization}

A one size fits all approach is simply inappropriate to cater to the wide range of abilities within the ID population. Potential areas for customization identified by the participants with ID therefore included the interaction modalities, background color, and artificial voice employed, which matched the findings of the experts in [14, 15]. Additionally, the end users requested the ability to switch between multiple styles of image sets used to display potential medical conditions, like that in [13].

\section{Identifiable Medical Imagery}

Finally, a variety of factors have been identified that affect the clarity of medical images - a resource shown to be crucial to the utilization of AAC technologies [14, 15, 23]. Some of these factors matched the findings made by Medhi et al. [42] when exploring text free interfaces. Both sets of participants gravitated towards the more real looking photographs. In addition, color was used to convey and distinguish between complex concepts (e.g. type of pain). Other factors that increased the clarity of medical images, included: displaying the extremity of emotions; capturing the natural body language conveyed by an individual; and avoiding overly graphic images

\section{LIMITATIONS \& FUTURE WORK}

The primary limitation of this study involves the demographics of the participants with mild ID. 9 of the 10 individuals came from, and attended consultations in, major cities that operate within a single healthcare system. Consequently, there is scope to explore the needs of rural patients and those from other countries, since their requirements may be significantly different. Further opportunities for future work include: building on the requirements extracted from the GPs in $[14,15]$ to further explore their communication and technological needs; designing an image set to be included in the app; and evaluating a concrete probe within the primary care context.

\section{CONCLUSION}

AAC technologies have the potential to assist people with mild ID throughout all aspects of life, yet our findings show their use remains limited within the clinical context. Just one of the ten participants regularly utilized an aid (patient passport) during consultations, despite the call for the embedment of high-tech AAC devices being made as far back as 1997 [43]. One reason for this may be the lack of guidelines to assist developers in creating such technologies. The authors could only identify those disseminated by Gibson et al. [14-16] yet their studies suffered from a lack of end-user involvement.

Consequently, we extracted design requirements for a clinical AAC application from ten people with mild ID. The participants believe that the aid can assist in mitigating barriers across the entire consultation process, beginning with reserving and accessing appointments. The primary method of promoting communication aligned with the views of the experts in [14-16], by supplying GPs with symptoms selected from an accessible questionnaire. This questionnaire should adapt to both the user's accessibility and medical needs and utilize evidence on the health trends experienced by people with ID. As such, commonly overlooked conditions may also be brought to the attention of the GP and potential time constraints may be alleviated. 


\section{REFERENCES}

1. Shanafelt, T.D., Hasan, O., Dyrbye, L.N., Sinsky, C., Satele, D., Sloan, J., West, C.P.: Changes in Burnout and Satisfaction With Work-Life Balance in Physicians and the General US Working Population Between 2011 and 2014. Mayo Clin. Proc. 90, 16001613

https://doi.org/10.1016/j.mayocp.2015.08.023

(2015).

2. Hall, L.H., Johnson, J., Watt, I., O'Connor, D.B.: Association of GP wellbeing and burnout with patient safety in UK primary care: a cross-sectional survey. Br J Gen Pract. 69, e507-e514 (2019). https://doi.org/10.3399/bjgp19X702713

3. Trollor, J.N., Ruffell, B., Tracy, J., Torr, J.J., Durvasula, S., Iacono, T., Eagleson, C., Lennox, N.: Intellectual disability health content within medical curriculum: an audit of what our future doctors are taught. BMC Med Educ. 16, (2016). https://doi.org/10.1186/s12909-016-0625-1

4. Hemsley, B., Balandin, S.: A Metasynthesis of Patient-Provider Communication in Hospital for Patients with Severe Communication Disabilities: Informing New Translational Research. Augment Altern Commun. 30, 329-343 (2014). https://doi.org/10.3109/07434618.2014.955614

5. Heslop, P., Blair, P., Fleming, P., Hoghton, M., Marriott, A., Russ, L.: Confidential Inquiry into premature deaths of people with learning disabilities, http://www.bristol.ac.uk/media-

library/sites/cipold/migrated/documents/fullfinalrepor t.pdf

6. Krahn, G.L., Hammond, L., Turner, A.: A cascade of disparities: health and health care access for people with intellectual disabilities. Ment Retard Dev Disabil Res Rev. 12, 70-82 (2006). https://doi.org/10.1002/mrdd.20098

7. Simpson, N.J.: Bridging primary and secondary care for people with learning disabilities. Advances in Psychiatric Treatment. 1, 207-213 (1995). https://doi.org/10.1192/apt.1.7.207

8. Phillips, A., Morrison, J., Davis, R.W.: General practitioners' educational needs in intellectual disability health. J Intellect Disabil Res. 48, 142-149 (2004)

9. Ali, A., Scior, K., Ratti, V., Strydom, A., King, M., Hassiotis, A.: Discrimination and Other Barriers to Accessing Health Care: Perspectives of Patients with Mild and Moderate Intellectual Disability and Their Carers. PLOS ONE. 8, e70855 (2013). https://doi.org/10.1371/journal.pone.0070855

10. Dodd, K., Brunker, J.: 'Feeling Poorly': Report of a Pilot Study Aimed to Increase the Ability of People with Learning Disabilities to Understand and Communicate About Physical Illness. British Journal of Learning Disabilities. 27, 10-15 (1999). https://doi.org/10.1111/j.1468-3156.1999.tb00076.x
11. Northway, R., Rees, S., Davies, M., Williams, S.: Hospital passports, patient safety and person-centred care: A review of documents currently used for people with intellectual disabilities in the UK. J Clin Nurs. 26, 5160-5168 (2017). https://doi.org/10.1111/jocn.14065

12. Prior, S.: HCI Methods for Including Adults with Disabilities in the Design of CHAMPION. In: CHI '10 Extended Abstracts on Human Factors in Computing Systems. pp. 2891-2894. ACM, New York, NY, USA (2010)

13. Prior, S., Waller, A., Kroll, T.: The CHAMPION Software Project. In: The Proceedings of the 13th International ACM SIGACCESS Conference on Computers and Accessibility. pp. 287-288. ACM, New York, NY, USA (2011)

14. Gibson, R.C., Bouamrane, M.-M., Dunlop, M.: Mobile support for adults with mild learning disabilities during clinical consultations. In: Proceedings of the 20th International Conference on Human-Computer Interaction with Mobile Devices and Services - MobileHCI '18. pp. 1-8. ACM Press, Barcelona, Spain (2018)

15. Gibson, R.C., Bouamrane, M.-M., Dunlop, M.: Design Requirements for a Digital Aid to Support Adults With Mild Learning Disabilities During Clinical Consultations: Qualitative Study With Experts. JMIR Rehabilitation and Assistive Technologies. 6, e10449 (2019). https://doi.org/10.2196/10449

16. Gibson, R.C., Bouamrane, M.-M., Dunlop, M.: Experts Views on the Use of Mobile Devices to Support Patients with Mild Learning Disabilities During Clinical Consultations. Stud Health Technol Inform. 264, 1199-1203 (2019). https://doi.org/10.3233/SHTI190416

17. Mirenda, P.: Toward Functional Augmentative and Alternative Communication for Students With Autism: Manual Signs, Graphic Symbols, and Voice Output Communication Aids. Lang Speech Hear Serv Sch. 34, 203-216 (2003). https://doi.org/10.1044/0161-1461(2003/017)

18. Menzies, R., Herron, D., Scott, L., Freeman, R., Waller, A.: Involving Clinical Staff in the Design of a Support Tool Improve Dental Communication for Patients with Intellectual Disabilities. In: Proceedings of the 15th International ACM SIGACCESS Conference on Computers and Accessibility. pp. 55:1-55:2. ACM, New York, NY, USA (2013)

19. WHO: Definition: intellectual disability, http://www.euro.who.int/en/healthtopics/noncommunicable-diseases/mentalhealth/news/news/2010/15/childrens-right-to-familylife/definition-intellectual-disability

20. Brodrick, D., Lewis, D., Worth, A., Marland, A.: One-page patient passport for people with learning 
disabilities. Nurs Stand. 25, 35-40 (2011). https://doi.org/10.7748/ns2011.07.25.47.35.c8644

21. Talking Mats Limited: Talking Mats - Improving communication, improving lives, https://www.talkingmats.com/

22. Bell, D.M., Cameron, L.: From Dare I say ... ? to I dare say: a case example illustrating the extension of the use of Talking Mats to people with learning disabilities who are able to speak well but unwilling to do so. British Journal of Learning Disabilities. 36, 122-127 (2008). https://doi.org/10.1111/j.14683156.2007.00475.x

23. Boström, P., Eriksson, E.: Design for Self-reporting Psychological Health in Children with Intellectual Disabilities. In: Proceedings of the 14th International Conference on Interaction Design and Children. pp. 279-282. ACM, New York, NY, USA (2015)

24. Boström, P., Johnels, J.Å., Thorson, M., Broberg, M.: Subjective Mental Health, Peer Relations, Family, and School Environment in Adolescents with Intellectual Developmental Disorder: A First Report of a New Questionnaire Administered on Tablet PCs. Journal of Mental Health Research in Intellectual Disabilities. $\quad 9, \quad 207-231 \quad$ (2016). https://doi.org/10.1080/19315864.2016.1186254

25. Boström, P., Broberg, M.: Protection and restriction: A mixed-methods study of self-reported well-being among youth with intellectual disabilities. JARID. 31, 164-176 https://doi.org/10.1111/jar.12364

(2018)

26. Craig, P., Dieppe, P., Macintyre, S., Michie, S., Nazareth, I., Petticrew, M.: Developing and evaluating complex interventions: the new Medical Research Council guidance. BMJ. 337, (2008). https://doi.org/10.1136/bmj.a1655

27. Bradshaw, J.: The use of augmentative and alternative communication apps for the iPad, iPod and iPhone: an overview of recent developments. Tizard Learning Disability Review. (2013). https://doi.org/10.1108/13595471311295996

28. Baxter, S., Enderby, P., Evans, P., Judge, S.: Barriers and facilitators to the use of high-technology augmentative and alternative communication devices: a systematic review and qualitative synthesis. Int $\mathrm{J}$ Lang Commun Disord. 47, 115-129 (2012). https://doi.org/10.1111/j.1460-6984.2011.00090.x

29. Alper, S., Raharinirina, S.: Assistive Technology for Individuals with Disabilities: A Review and Synthesis of the Literature. J Spec Educ Technol. 21, 47-64 (2006) https://doi.org/10.1177/016264340602100204

30. Blackstone, S.W., Williams, M.B., Joyce, M.: Future AAC technology needs: consumer perspectives. Assist Technol. 14, 3-16 (2002). https://doi.org/10.1080/10400435.2002.10132051

31. Horner-Johnson, W., Bailey, D.: Assessing Understanding and Obtaining Consent from Adults with Intellectual Disabilities for a Health Promotion Study. J Policy Pract Intellect Disabil. 10, (2013). https://doi.org/10.1111/jppi.12048

32. Doyle, J.: Using Focus Groups as a Research Method in Intellectual Disability Research: A Practical Guide, http://www.fedvol.ie/_fileupload/Research/focus $\% 20$ groups\%20a\%20practical\%20guide.pdf, (2009)

33. Zisook, M., Patel, R.: Exploring the Communication Needs of People with Disabilities Through Participatory Design. In: CHI'14. p. 4. Association for Computing Machinery (2014)

34. Sitbon, L., Farhin, S.: Co-designing interactive applications with adults with intellectual disability. In: Proceedings of the 29th Australian Conference on Computer-Human Interaction. pp. 487-491. ACM, Brisbane, Queensland, Australia (2017)

35. Gale, N.K., Heath, G., Cameron, E., Rashid, S., Redwood, S.: Using the framework method for the analysis of qualitative data in multi-disciplinary health research. BMC Medical Research Methodology. 13, $117 \quad$ (2013). https://doi.org/10.1186/1471-2288-13-117

36. GAIN: Guidelines on Caring For People with a Learning Disability in General Hospital Settings, https://rqia.org.uk/RQIA/files/81/81662c46-b7bb43a5-9496-a7f2d919c2a3.pdf

37. Charlop-Christy, M.H., Carpenter, M., Le, L., LeBlanc, L.A., Kellet, K.: Using the picture exchange communication system (PECS) with children with autism: assessment of PECS acquisition, speech, social-communicative behavior, and problem behavior. J Appl Behav Anal. 35, 213-231 (2002). https://doi.org/10.1901/jaba.2002.35-213

38. Gibson, R.C., Bouamrane, M.-M., Dunlop, M.D.: Ontology-Driven, Adaptive, Medical Questionnaires for Patients with Mild Learning Disabilities. In: Bramer, M. and Petridis, M. (eds.) Artificial Intelligence XXXVI. pp. 107-121. Springer International Publishing, Cham (2019)

39. Stein, J.: What is Developmental Dyslexia? Brain $\begin{array}{llll}\text { Sciences. } & 8, & 26 & \text { (2018). }\end{array}$ https://doi.org/10.3390/brainsci8020026

40. Lennox, N.G., Diggens, J.N., Ugoni, A.M.: The general practice care of people with intellectual disability: barriers and solutions. J Intellect Disabil Res. 41 ( Pt 5), 380-390 (1997)

41. Pelleboer-Gunnink, H.A., Oorsouw, W.M.W.J.V., Weeghel, J.V., Embregts, P.J.C.M.: Mainstream health professionals' stigmatising attitudes towards people with intellectual disabilities: a systematic review. Journal of Intellectual Disability Research. 61, 411-434 (2017). https://doi.org/10.1111/jir.12353

42. Medhi, I., Sagar, A., Toyama, K.: Text-Free User Interfaces for Illiterate and Semiliterate Users. Information Technologies and International Development. 4, 14 (2007) 
43. Jones, R.G., Kerr, M.P.: A randomized control trial of an opportunistic health screening tool in primary care for people with intellectual disability. J Intellect Disabil Res. 41 ( Pt 5), 409-415 (1997) 